\title{
Segmentação do lúmen em imagens de IOCT usando Fuzzy Connectedness e Reconstrução Binária Morfológica
}

\author{
Diego Armando Cardona Cardenas*, Matheus Cardoso Moraes, Sérgio Shiguemi Furuie
}

Resumo No ano 2010, doenças cardiovasculares (CVD) causaram 33\% do total das mortes no Brasil. Tomografia Ótica Coerente Intravascular (IOCT) é uma tecnologia que oferece imagens in vivo para detecção e monitoramento da progressão de CVD. O exame de IOCT permite mais precisão no diagnóstico; contudo, ainda é pequena a variedade de métodos quantitativos aplicados a IOCT na literatura, em comparação à outras modalidades relacionadas. Portanto neste trabalho é proposto um método de segmentação do lúmen, baseado em uma combinação de Fuzzy Connectedness, com múltiplas funções de afinidade, e Operações Morfológicas. As funções de afinidade usadas neste trabalho são: (I) Clássica, (II) Pesos Dinâmicos e (III) Bhattacharyya. Esta última é baseada no coeficiente de Bhattacharyya, utilizado habitualmente para speckle tracking. Primeiro, características não desejadas da imagem são atenuadas. Depois, informações da parede do vaso são obtidas utilizando Fuzzy Connectedness e um processo de binarização dinâmico. Finalmente, operações morfológicas são realizadas para melhorar o lúmen segmentado. Para avaliar o método proposto, um conjunto de 130 imagens advindas de humanos, porcos, e coelhos foram segmentadas e comparadas com seus respectivos "Gold Standards" feitos por especialistas. Uma média de verdadeiros positivos (TP\%) =98,08 e de falsos positivos $(\mathrm{FP} \%)=2,34$ foram obtidas. Com isso, o método proposto resultou em uma maior eficácia do que os estudos publicados anteriormente, encorajando seu uso.

Palavras-chave Tomografia ótica coerente intravascular, Segmentação, Fuzzy connectedness, Bhattacharyya, Operações morfológicas.

\section{Lumen Segmentation in IOCT images using Fuzzy and Binary Morphological Reconstruction}

\begin{abstract}
In 2010 cardiovascular disease (CVD) caused 33\% of the total deaths in Brazil. Intravascular Optical Coherent Tomography (IOCT) is an imaging technology that provides in vivo detection and monitoring of the progression of coronary heart disease. IOCT exam allows more accurate diagnoses; nonetheless, the set of quantitative methods applied to IOCT in the literature is small compared to other related modalities. Therefore, the proposed approach presents a lumen segmentation method, based on a combination of Fuzzy Connectedness, with multiple affinity functions, and Morphological Operations. The affinity functions used in this work are: (I) classical, (II) Dynamic weights (III) Bhattacharyya. The latter is based on the Bhattacharyy a coefficient, commonly used for speckle tracking. Firstly, unwanted features of the image are attenuated. Then, vessel-wall information is obtained using Fuzzy Connectedness and dynamic binarization process. Finally, morphological operations are performed to improve the segmented lumen. To evaluate the proposed method, a set of 130 images from humans, pigs and rabbits were segmented and compared to their corresponding gold standard made by experts. An average of true positive (TP\%) =98.08, and false positive $(F P \%)=2.34$ were obtained. Hence, the use of the proposed method is suggested since it has yielded higher efficiency than previously published studies.
\end{abstract}

Keywords Intravascular Optical Coherent Tomography(IOCT), Segmentation, Fuzzy connectedness, Bhattacharyya, Morphological operations. 


\section{Introdução}

De acordo com a Organização Mundial da Saúde (WHO), estima-se que no ano 2008, mais de 17 milhões de pessoas morreram de doenças cardiovasculares (CVD), e acima de $80 \%$ dessas mortes ocorreram em países de baixa ou média renda (World..., 2011a). No Brasil as mortes por CVD em 2010, corresponderam a 33\% das mortes (World..., 2011b).

$\mathrm{O}$ acúmulo de placas, calcificadas, lipídicas e fibrosas, em vasos sanguíneos é chamada aterosclerose e é uma das principais causas da maioria das internações por doenças coronárias (Meng et al., 2007). A aterosclerose pode causar o estreitamento das artérias, dificultando a irrigação sanguínea das áreas afetadas, levando à tromboses, acidentes vasculares cerebrais, infarto do miocárdio, ou morte cardíaca súbita (Barajas et al., 2007; Pollock e Wilmore, 1993). O risco da doença aumenta com alguns hábitos, como por exemplo, sedentarismo, alimentação inadequada, tabagismo ou histórico familiar de doenças cardíacas. No entanto, algumas pessoas com aterosclerose não apresentam sinais ou sintomas, e as causas da doença não são sempre conhecidas (Pollock e Wilmore, 1993). A implantação de stents é o método mais comum para tratar pacientes com obstrução nas coronárias (Souza et al., 2004). Contudo, esta metodologia pode causar traumatismo na parede do vaso, desencadeando uma re-estenose, a qual é o crescimento de uma nova íntima pela migração de células musculares lisas da camada média para a íntima, e a posterior multiplicação destas células (Costa e Fagundes, 2002). O contínuo crescimento da íntima (neo intima) pode causar estreitamento do vaso, causando novamente problemas de irrigação do coração. Assim, tecnologias que possam auxiliar os profissionais da saúde para melhores diagnósticos e terapias são sempre importantes.

Algumas tecnologias de geração de imagens oferecem alternativas para a detecção in vivo e monitoramento da progressão das doenças coronárias (Meng et al., 2007). Tomografia Ótica Coerente Intravascular (IOCT) é uma nova técnica de imagem médica que vem ganhando importância. Ela mede a intensidade das reflexões retro-espalhadas de luz infravermelha para gerar imagens tomográficas em tempo real (Unal et al., 2010). Apesar de IOCT ter baixa penetração através do sangue e dos tecidos (Puri et al., 2011), ele possui uma resolução de $10 \mu \mathrm{m}$, a qual é muito melhor em comparação com a resolução do Ultrassom intravascular (IVUS) (100-150 $\mu \mathrm{m})$ (Bouma et al., 2003), permitindo detectar e caracterizar melhor a composição da placa aterosclerótica (Rieber et al., 2006).

O princípio básico da geração de imagens IOCT pode ser visto na Figura 1. A partir de um interferômetro de Michelson, uma fonte de luz infravermelha é transmitida através de um divisor ótico, o qual gera um feixe de referência, refletido por um espelho, e um feixe de amostra que é refletido de acordo com a dispersão das diferentes camadas dos tecidos (Rubinstein et al., 2009). Os feixes refletidos são comparados e se os dois concordam no tempo, é gerado o fenômeno de interferência que é capturado pelo detector. Como a distância do espelho é conhecida, é possível determinar a distância das camadas dos tecidos pelo fenômeno de interferência. As diferentes distâncias obtidas são dispostas graficamente, tendo assim uma

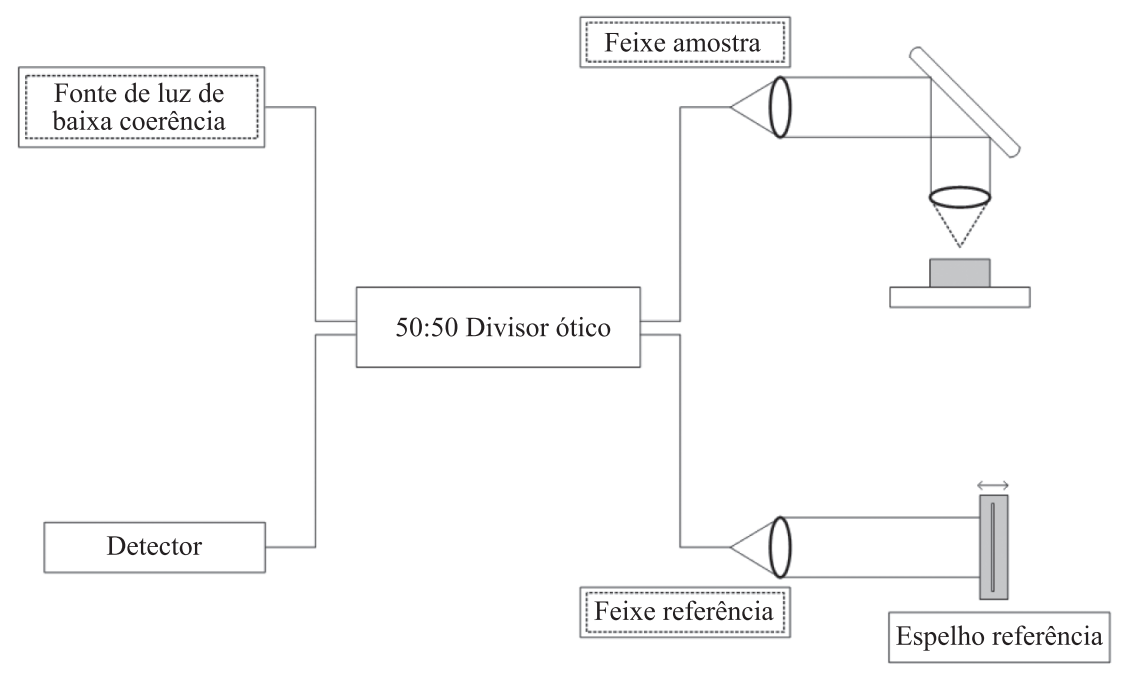

Figura 1. Esquema OCT com um interferômetro de Michelson (Brezinski, 2006).

Figure 1. Block diagram of OCT, with a Michelson interferometer (Brezinski, 2006). 
imagem em sentido axial (A-scan). A contínua geração de A-scans e um alinhamento adequado permite gerar uma imagem bidimensional (tomográfica) (Griñó et al., 2008). Nos equipamentos atuais de IOCT, as imagens são adquiridas pela introdução de seu cateter na artéria coronária, seguido por um movimento de retirada. Enquanto removido do vaso, o cateter executa movimentos de rotação emitindo luz radialmente ao tecido vascular, e assim, obtendo um fluxo contínuo de informações. No entanto, para que informações mais precisas a respeito dos vasos possam ser extraídas, é necessário segmentar essas imagens.

Segmentação: Segmentação de imagens é um processo no qual uma imagem é particionada em regiões distintas para identificar e delimitar objetos (Udupa et al., 2006). A segmentação pode ser feita com uma ampla quantidade de métodos, mas a escolha do melhor método depende das características da imagem e do problema a ser resolvido (Dougherty, 2009). As técnicas de segmentações podem ser divididas em 3 diferentes maneiras:

- Manualmente: um operador faz o delineamento manual das regiões de interesse;

- Semi-Automaticamente: um operador demarca alguns pontos (sementes) ou faz um contorno aproximado na área de interesse e um algoritmo finaliza a segmentação; e

- Automaticamente: um software faz todo o trabalho de segmentação, o operador ou médico só tem o trabalho de indicar as imagens a serem segmentadas.

Fuzzy Connectedness: Método de segmentação semiautomático, baseado em crescimento por regiões (Udupa e Samarasekera, 1996). O crescimento vai depender das características entre os pixels semente e os pixels da imagem, ou seja, a partir das sementes o algoritmo começa a relacionar quais pixels pertencem ao objeto. Essa relação é quantificada com valores entre 0 e 1 , indicando o grau de pertinência do pixel, quanto mais perto de 1, maior possibilidade tem o pixel de ser parte do objeto (Souza, 2010). Fuzzy Connectedness tem relações locais e globais para estabelecer o grau de pertinência dos pixels. A primeira é chamada relação de afinidade, e associa à cada par de pixels (c, d) um valor entre 0 e 1 , onde esse valor indica a similaridade entre 2 pixels. Na versão original da técnica, introduzida por Udupa e Samarasekera (1996), os autores propõe essa relação como uma combinação de relações locais de proximidade, similaridade e a homogeneidade, mas estabelece que mais relações de afinidade podem ser propostas. A afinidade pode ser definida como (Udupa e Samarasekera, 1996):

$$
\mu_{k}(c, d)=\mu_{\alpha}(c, d)\left[w_{1}^{*} \mu_{\Psi}(c, d)+w_{2}{ }^{*} \mu_{\phi}(c, d)\right]
$$

nos quais $\mathrm{w}_{1}$ e $\mathrm{w}_{2}$ são pesos que satisfazem

$$
w_{1}+w_{2}=1
$$

e $\mu_{\alpha}, \mu_{\psi}$ e $\mu_{\phi}$ são medidas de adjacência, Homogeneidade e Intensidade, respectivamente (Udupa e Samarasekera, 1996), obtidas por:

$$
\begin{aligned}
& \mu_{\alpha}(c, d)= \begin{cases}1, & \text { se } \sqrt{\sum_{i}\left(C_{i}-d_{i}\right)^{2}} \leq 1 \\
0, & \text { em outro caso }\end{cases} \\
& \mu_{\Psi}(c, d)=e^{-\frac{1}{2}\left(\frac{f(c)-f(d) \mid-m_{1}}{s_{1}}\right)^{2}}
\end{aligned}
$$

$\mu_{\phi}(c, d)=e^{-\frac{1}{2}\left(\frac{(0.5 *[f(c)+f(d)])-m_{2}}{s_{2}}\right)^{2}}$

os quais, $m_{l}$ e $s_{l}$ são a média e o desvio padrão das homogeneidades dos objetos e, $m_{2}$ e $s_{2}$ são a média e o desvio padrão de intensidades locais dos objetos.

A relação global é chamada de conectividade. Ela é baseada no caminho mais forte entre 2 pixels (maximização) e a força do caminho é medida como a menor afinidade encontrada em um caminho (Souza, 2010). Esta relação é dada por:

$\mu_{K}=\max _{p}\left(\min _{1<i<N_{p}}\left(\mu_{k}\left(s_{i-1}, s_{i}\right)\right)\right)$

para $N_{p}$ sendo o número de pixels entre 'c' e 'd', e $s_{i}$ e $s_{i-1}$ sendo vizinhos para um determinado caminho $p$. A saída do algoritmo é uma imagem de custos de conectividade que deve ser Binarizada a partir de um limiar, e assim obter o objeto segmentado (Souza, 2010).

Bhattacharyya: O coeficiente de Bhattacharyya é uma medida estatística de afinidade a qual mede a divergência de duas populações correlatas normais com os mesmos conjuntos de variâncias e covariâncias (Bhattacharyya, 1943). Ele é uma medida geométrica e fornece o cosseno entre dois vetores n-dimensionais. Quanto mais próximo o coeficiente for de 1, mais similares são os vetores.

Sejam duas populações multinomiais $s(i)$ e $p(i)$ as quais tem $\mathrm{N}$ classes com probabilidades associadas $s(i=1), \ldots, s(i=N)$ e $p(i=1), \ldots, p(i=N)$, respectivamente. Dado que $s(i)$ e $p(i)$ representam distribuições de probabilidade $\sum_{i}^{N} s(i)=\sum_{i}^{N} p(i)=1$, o coeficiente de Bhattacharyya pode se definir por (Aherne et al., 1998):

$$
\rho(s, p)=\sum_{i=1}^{N} \sqrt{s(i) \cdot p(i)}
$$

Bhattacharyya como Função de Afinidade: A função de afinidade Bhattacharyya é definida para um par de pixels (c, d) como:

$\mu_{k}(c, d, s)=\left[\rho\left(h_{s}, h_{c d}\right)\right]$ 
onde $h_{\mathrm{s}}$ é o histograma ponderado e normalizado do pixel semente $s$ e $h_{\mathrm{cd}}$ é a media dos histogramas ponderados e normalizados, $h_{\mathrm{c}}$ e $h_{\mathrm{d}}$, dos pixels $c$ e $d$ respectivamente, definido como

$h_{c d}(i)=\frac{h_{c}(i)+h_{d}(i)}{2}$

Para obter os histogramas é necessário estabelecer um kernel circular de raio $R$, onde cada valor estará ponderado com respeito ao inverso da distância euclidiana entre uma posição $p$, dentro do kernel, e o centro $c$ (Figura $2 \mathrm{~b}$-e), assim

$$
K_{c p}=\left\{\begin{array}{l}
\frac{1}{\text { dist }_{c p}} \text { se } c \neq p \text { e e dist } t_{c p} \leq R \\
1 \quad \text { se } c=p \\
0 \quad \text { se dist } t_{c p}>R
\end{array}\right.
$$

Atualmente alguns enfoques para a segmentação do Lúmen em imagens de IOCT, usando diferentes técnicas, foram propostos na literatura. No trabalho de Tung et al. (2011), os autores fazem a segmentação do lúmen da coronária utilizando uma combinação de um algoritmo de maximização de Expectativa (Dempster et al., 1977), um algoritmo Graph Cuts (Boykov e Jolly, 2001) e contornos ativos (Kass et al., 1988). No método proposto por Gurmeric et al. (2009), os pesquisadores utilizam contornos ativos que se propagam com equação diferencial ordinária (EDO) para atingir uma ótima solução. Dubuisson et al. (2009) propõem um método no qual combina binarização por Otsu (1979), segmentação morfológica e contornos ativos. Na técnica proposta por Sihan et al. (2008), é empregado um pré-processamento com o filtro gaussiano e operações morfológicas para tirar o lúmen, um filtro de Canny (Canny, 1986) para detectar bordas e um filtro baseado em threshold para tirar as bordas que não pertencem ao lúmen. No artigo de Tsantis et al. (2012), é aplicado um pré-processamento com a transformada de Hough e um modelo de Campos Aleatórios de Markov (Besag, 1986) para obter o lúmen em artéria femoral.

Apesar dos esforços, a segmentação da Coronária ainda é um problema, pois manualmente torna-se uma tarefa cansativa e demorada, e por ser uma modalidade nova em relação ao IVUS, muitas técnicas que apresentam excelentes desempenhos em outras modalidades, ainda não foram investigadas para IOCT. Uma dessas técnicas é o método Fuzzy Connectedness, o qual apresenta bons resultados na segmentação em outras modalidades (Ciesielski et al., 2007; Moonis et al., 2002; Udupa e Saha, 2003; Zaidi e Naqa, 2010), mas ainda não se conhece nenhum trabalho em que esta seja direcionada para a segmentação do lúmen em imagens de IOCT.

Adicionalmente, nenhum trabalho usando Bhattacharyya como função de afinidade Fuzzy foi encontrado até o momento. Bhattacharyya pode vir a ser uma excelente opção de função de afinidade para segmentação de imagens IOCT, pois apresenta alto desempenho para "Speckle tracking". Portanto, o objetivo deste trabalho é implementar e investigar a acurácia de um novo método de segmentação do lúmen em imagens IOCT baseado em Fuzzy Connectedness para três relações de afinidade, (i) clássica, (ii) Pesos dinâmicos, e (iii) Bhattacharyya.

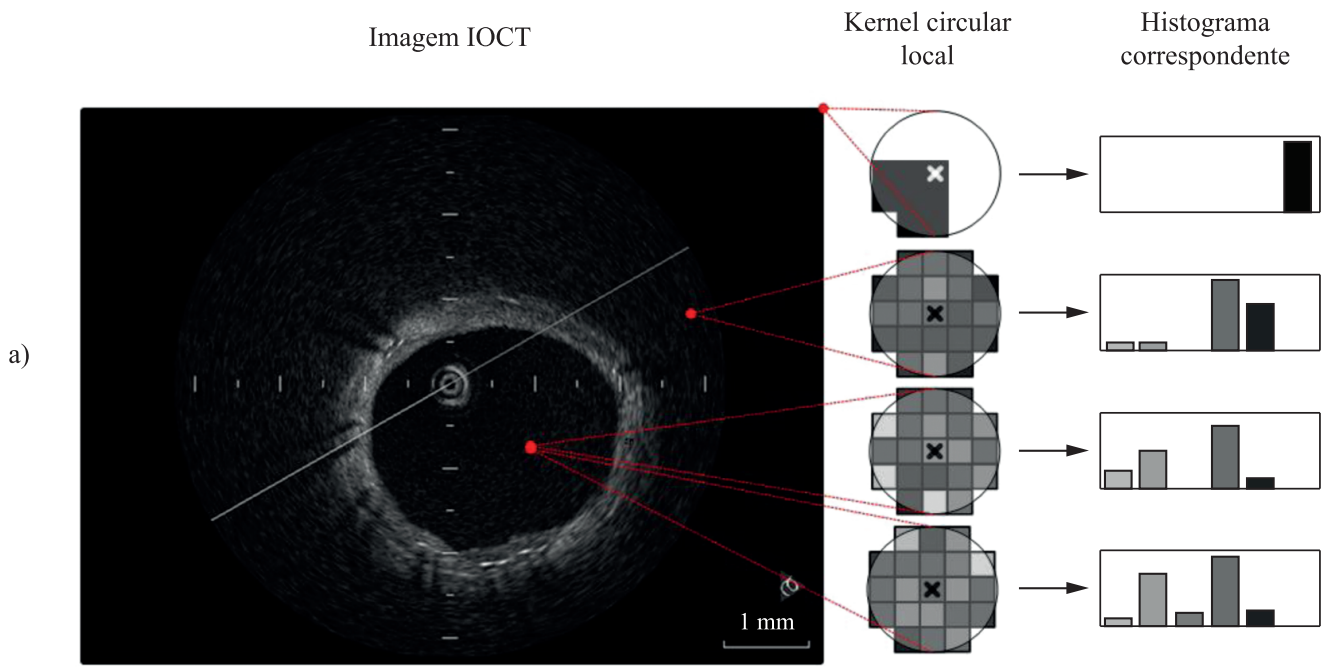

Figura 2. a) Imagem característica de IOCT. (b), (c), (d) e (e) Ilustração do kernel circular local em diferentes posições, e seus respectivos histogramas.

Figure 2. a) IOCT image. (b), (c), (d) and (e) Illustration of the local circular kernel in different positions, and their corresponding histograms. 


\section{Materiais e Métodos}

Neste estudo é feita a segmentação semi-automática do lúmen de um conjunto de 130 imagens da Coronária de diferentes tamanhos, forma e posição espacial. As imagens são advindas de porcos, coelhos e humanos, cedidas pelo Instituto do Coração do Hospital das Clínicas da Faculdade de Medicina da Universidade de São Paulo (InCor). A avaliação foi feita comparando as imagens segmentadas com seus respectivos "gold standards", feitos manualmente por especialistas, e comparando os resultados adquiridos, com os de estudos publicados. Para cada tipo de relação de afinidade utilizada, a metodologia pode ser dividida em três etapas. Na primeira etapa, Pré-processamento, são normalizadas as imagens, e as características não desejadas, como o cateter, são atenuadas. Na etapa seguinte, Processamento, são extraídas informações da parede do vaso por meio da utilização de Fuzzy connectedness e binarização por "threshold". Na etapa final, Pós-processamento, um teste de "Branch Opening" e um conjunto de operações morfológicas é realizado para melhorar o lúmen segmentado. Para a execução das etapas foi utilizada a linguagem Java no ambiente Imaje-J.

\section{Pré-processamento}

No pré-processamento, primeiramente a imagem original é normalizada a um tamanho de $400 \times 400$, $\boldsymbol{I}_{\text {normalizada }}$ (Figura 3a). Posteriormente, para eliminar os efeitos não desejados do reflexo do cateter, as intensidades dos pixels na área do transdutor são alteradas, gerando uma imagem auxiliar $I_{\text {sem cateter }}$. A seguinte equação define a atenuação do reflexo:

$$
I_{\text {sem cateter }}(x, y)=\left\{\begin{array}{l}
\mu, \quad \text { se raio }<12 \text { pixels } \\
I_{\text {normalizada }}(x, y), \text { em outro caso }
\end{array}\right.
$$

os quais, $\mu$ é a intensidade do pixel semente necessário para o método Fuzzy Connectedness, raio é o distância entre o pixel atual e o centro da imagem, e $\boldsymbol{I}_{n}$ é a intensidade da imagem original normalizada. No caso da utilização da afinidade baseada no coeficiente de Bhattacharyya, são calculados e armazenados numa matriz os histogramas normalizados para todos os pixels pertencentes a $\boldsymbol{I}_{\text {normalizada }}$, empregando para este, um kernel circular de raio $\mathrm{R}=5$. Os histogramas pertencentes à área do transdutor são modificados para serem iguais ao histograma da semente, assim:

$M_{\text {hist }}(x, y)=h_{s}$, se raio $(x, y)<12$ spels

para $M_{\text {hist }}$ sendo a matriz de histogramas normalizados, $h_{s}$ o histograma normalizado do spel semente, e raio é o raio entre o pixel atual e o centro da imagem.

\section{Processamento}

No Processamento, partindo da imagem pré-processada $I_{\text {sem cateter }}$, ou da matriz de histogramas $M_{\text {hist }}$, dependendo da relação de afinidade utilizada, se obtém uma imagem binarizada do lúmen por meio da realização de duas operações combinadas, Fuzzy Connectedness e um novo método de binarização. Primeiramente, para os métodos que utilizam relações de afinidade "clássica", Equação 1, mantendo a condição da Equação 2, onde $w_{1}+w_{2}=1$, são definidas duas relações de afinidade. Na primeira, os pesos $\mathrm{w}_{1}$ e $\mathrm{w}_{2}$ são iguais, ou seja,

$w_{1}=w_{2}=0,5$

e na segunda função de afinidade, o calculo dos pesos $w_{1}$ e $w_{2}$ é dinâmico (Pednekar e Kakadiaris, 2006), usando:

$\mathrm{w}_{1}=\frac{\mu_{\phi}(\mathrm{c}, \mathrm{d})}{\mu_{\phi}(\mathrm{c}, \mathrm{d})+\mu_{\Psi}(\mathrm{c}, \mathrm{d})}$

para $\mu_{\psi}$ e $\psi_{\varphi}$ sendo as funções de Homogeneidade e Intensidade definidas nas Equações 3 e 4 , e $w_{2}=1-w_{1}$. Além das relações acima, a afinidade Bhattacharyya definida na Equação 8, também é empregada.

O uso do fuzzy connectedness, utilizando qualquer relação de afinidade, vai gerar uma imagem de custo

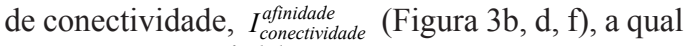
é binarizada, I bininizade $^{\text {afizada }}$ (Figura 3c, e, g) usando a seguinte equação:

$I_{\text {binarizada }}^{\text {afinidade }}=\left\{\begin{array}{l}0, \text { se } I_{\text {conectividade }}^{\text {afinidade }}<\frac{m_{3}+x}{2} \\ 1, \text { se } I_{\text {conectividade }}^{\text {afinidade }} \geq \frac{m_{3}+x}{2}\end{array}\right.$

Nos quais $m_{3}$ é a média da $I_{\text {conectividade }}^{\text {afinidade }}$, e $x$ é o valor da conectividade do pixel semente.

\section{Pós-processamento}

Como a qualidade do objeto binarizado, depende das características da coronária na imagem original, da relação de afinidade utilizada e do "threshold" da binarização, as informações extraídas e perdidas tem que ser melhoradas e estimadas, respectivamente. Assim, no Pós-processamento é utilizado uma seqüência de operações morfológicas, chamada de reconstrução binária morfológica [UMB], para melhorar e estimar informações do objeto extraído anteriormente.

Teste de "Branch Opening" - "Branch Opening" são aberturas localizadas na parede da coronária em algumas imagens de IOCT, devido à aquisição em regiões de bifurcação. Por causa dessas aberturas, um caminho conectando o lúmen à adventícia é criado, fazendo com que a conectividade seja maior nos pixels pertencentes à adventícia, obtendo, depois da binarização, um objeto muito maior que apenas com 
o lúmen (Figura 5b). Devido à essa característica, primeiro se faz um teste de "Branch Opening", o qual consiste em calcular a porcentagem de pixels brancos dentro da $I_{\text {binarizadda }}^{\text {afinide }}$. Se essa porcentagem for abaixo de um limiar, conclui-se que a imagem não tem "Branch Opening", pois só o lúmen estará binário, caso contrário ela tem "Branch Opening". Esse limiar foi definido empiricamente em 17,5\%. Consequentemente, duas seqüências de operações morfológicas, foram projetadas para cada um dos casos, uma seqüência é chamada Imagem sem "Branch Opening", e a outra Imagem com "Branch Opening", todas as operações, e possíveis variações devido à função de afinidade, estão resumidas na Tabela 1, e serão explicadas abaixo usando imagem binarizada $I_{\text {binarizada }}^{w}$ iguais advinda da utilização da afinidade com pesos iguais.

Imagem sem "Branch Opening" - No caso da imagem I Ifinidade "izada não possuir "Branch Opening", a seguinte seqüência de operações morfológicas são usadas. Com a finalidade de tirar os ruídos pequenos, pontos pretos (Figura $4 \mathrm{a}$ ), trazidos devido às características da imagem original de IOCT e do processo de binarização, primeiramente, sobre a

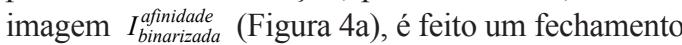
(Gonzalez e Woods, 2008), (Tabela 1 - linha 1),
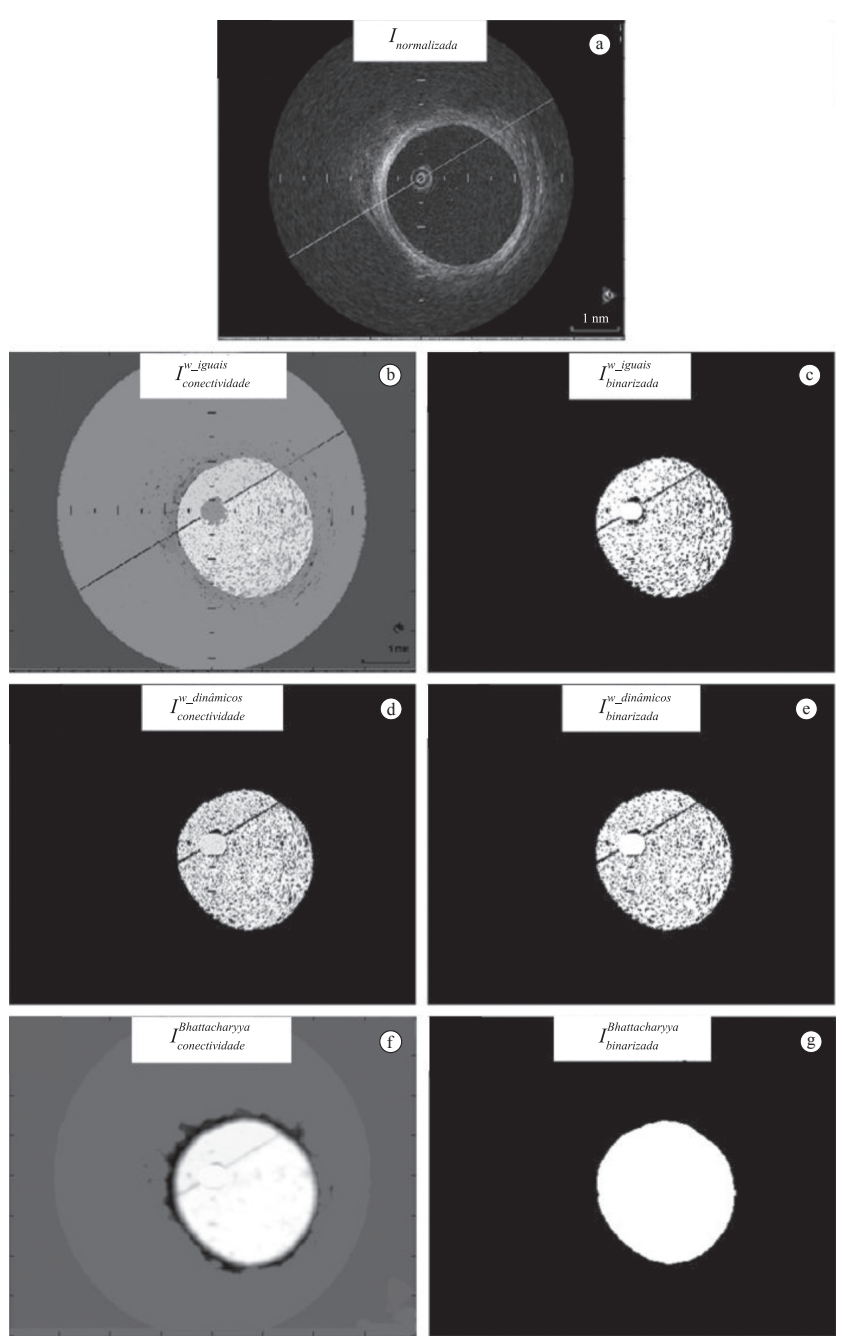

Figura 3. a) $I_{\text {normalizada }}$, é a imagem normalizada. b) $I_{\text {conectividade }}^{w}$ é a imagem de valores de conectividade para pesos $\mathrm{w}_{1}$ e $\mathrm{w}_{2}$ iguais.

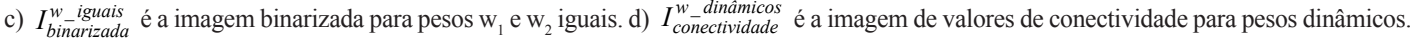

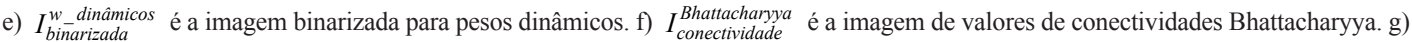
$I_{\text {binarizada }}^{\text {Bhatacha }}$ é a imagem binarizada para Bhattacharyya.

Figure 3. a) $I_{\text {normalizada }}$, the normalized image. b) $I_{\text {conectividade }}^{w \text { iguais }}$ image of connectivity for weights with the same value. c) $I_{\text {binarizada }}^{w}$, binary image for weights with the same value. d) $I_{\text {conectividade }}^{w}$, image of connectivity for dynamic weights. e) $I_{\text {binarizada }}^{w}$ dinâm binary image for dynamic

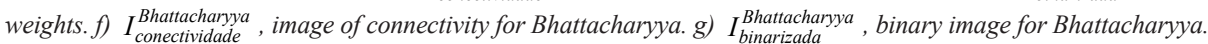


com um elemento estruturante circular de raio $3, S_{3}$, é feito uma operação de preenchimento (Gonzalez e resultando em a imagem auxiliar $I_{\text {fechada } 3}$ (Figura 4b). Woods, 2008), (Tabela 1 - linha 2), assegurando que Posteriormente sobre esta imagem, $I_{\text {fechada__ }}($ Figura $4 \mathrm{~b}$ ), possíveis ruídos remanescentes sejam retirados, e
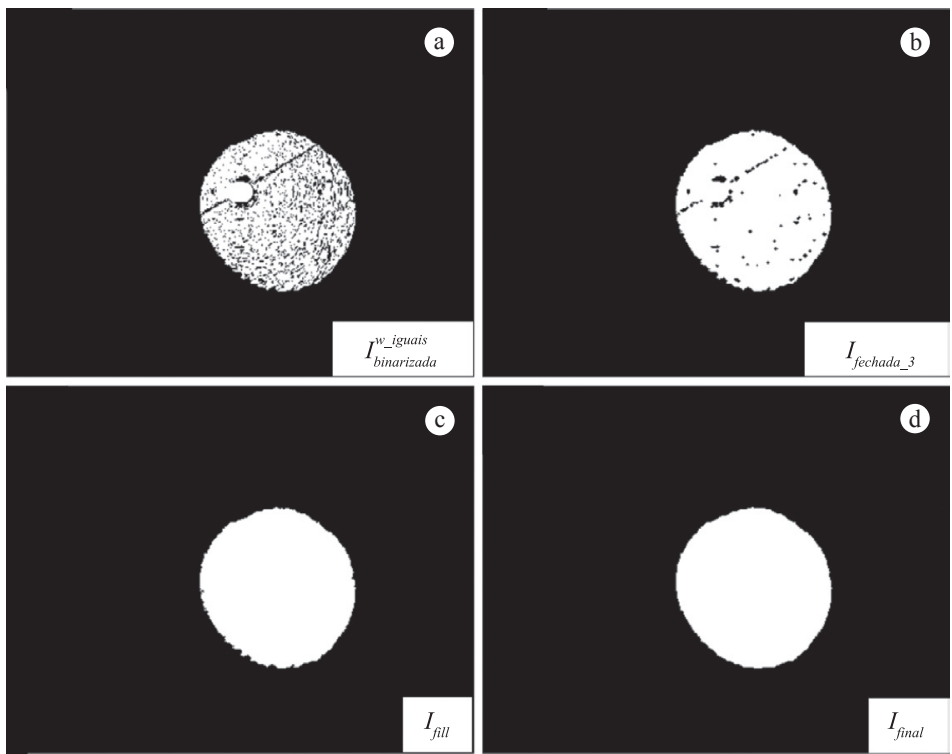

Figura 4. a) Imagem binarizada para pesos iguais. b) Imagem binarizada fechada com kernel de raio $R=3$. c) Imagem fechada preenchida. d) Imagem final após do fechamento da imagem preenchida com kernel de raio dinâmico $\mathrm{R}_{\text {din }}$.

Figure 4. a) Binary image for weights with the same value. b) Binary image after a closing procedure with kernel of radius $R=3$. c) Image after a closing and filling procedure. d) Final image after another closing and filling procedure with kernel of dynamic radius $R_{\text {din }}$

Tabela 1. Resumo das operações feitas para Pós-processamento.

Table 1. Summary of the Pos-processing operations.

\begin{tabular}{|c|c|c|c|c|c|c|c|}
\hline \multirow[b]{2}{*}{$\begin{array}{l}\text { "Branch } \\
\text { Opening" }\end{array}$} & \multirow[b]{2}{*}{ Linha } & \multirow[b]{2}{*}{ Operação } & \multirow[b]{2}{*}{ Equação } & \multirow[b]{2}{*}{ Raio } & \multicolumn{3}{|c|}{ Afinidade } \\
\hline & & & & & $\begin{array}{l}\text { Pesos } \\
\text { iguais }\end{array}$ & $\begin{array}{c}\text { Pesos } \\
\text { dinâmicos }\end{array}$ & Bhattacharyya \\
\hline \multirow{3}{*}{ Sem } & 1 & Fechamento & $I_{\text {fechada }}=I_{x} \cdot S_{\text {raio }}$ & 3 & $\checkmark$ & $\checkmark$ & $\checkmark$ \\
\hline & 2 & Fill & 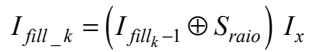 & 1 & $\checkmark$ & $\checkmark$ & $\checkmark$ \\
\hline & 3 & Fechamento & $I_{\text {fechada }}=I_{x} \cdot S_{\text {raio }}$ & $\mathrm{R}_{\text {din }}$ & $\checkmark$ & $\checkmark$ & $\checkmark$ \\
\hline \multirow{10}{*}{ Com } & 4 & Fechamento & $I_{\text {fechada }}=I_{x} \cdot S_{\text {raio }}$ & 5 & $\checkmark$ & $\checkmark$ & $\checkmark$ \\
\hline & 5 & Abertura & $I_{\text {aberta }}=I_{x} \circ S_{\text {raio }}$ & 3 & & $\checkmark$ & \\
\hline & 6 & Polar invertida & & - & $\checkmark$ & $\checkmark$ & $\checkmark$ \\
\hline & 7 & Fechamento & $I_{\text {fechada }}=I_{x} \cdot S_{\text {raio }}$ & 9 & $\checkmark$ & $\checkmark$ & $\checkmark$ \\
\hline & 8 & Abertura & $I_{\text {aberta }}=I_{x} \circ S_{\text {raio }}$ & 12 & $\checkmark$ & $\checkmark$ & $\checkmark$ \\
\hline & 9 & Preenchimento & $I_{\text {down }}=\operatorname{Fill}_{\text {down }}\left(I_{\text {polar_}_{-} X}\right)$ & - & $\checkmark$ & $\checkmark$ & $\checkmark$ \\
\hline & 10 & Interpolação & & - & $\checkmark$ & $\checkmark$ & $\checkmark$ \\
\hline & 11 & Abertura & $I_{\text {aberta }}=I_{x} \circ S_{\text {raio }}$ & 25 & & $\checkmark$ & \\
\hline & 12 & $\begin{array}{l}\text { Cartesiana } \\
\text { invertida }\end{array}$ & & - & $\checkmark$ & $\checkmark$ & $\checkmark$ \\
\hline & 13 & Fechamento & $I_{\text {fechada }}=I_{x} \cdot S_{\text {raio }}$ & $\mathrm{R}_{d i n}$ & $\checkmark$ & $\checkmark$ & $\checkmark$ \\
\hline
\end{tabular}


assim gerando a imagem auxiliar $I_{\text {fill }}$ (Figura 4c). Por último, para melhorar as bordas do objeto binarizado e segmentado na imagem $I_{\text {fill }}$ (Figura $4 \mathrm{c}$ ) é feito um fechamento, (Tabela 1 - linha 3), com um elemento estruturante circular, cujo raio é ajustado dinamicamente, $R_{d i n}$, proporcional ao menor raio do objeto (Moraes e Furuie, 2011), gerando assim a imagem $I_{\text {frul }}$ (Figura 4d).
Imagem com "Branch Opening" - Se a imagem original normalizada $I_{\text {normalizada }}$ (Figura 5a) possuir "Branch Opening", não só o lúmen, mas também as informações da adventícia serão extraídas depois do processamento $I_{\text {binarizadada }}^{\text {afindalo }}$ (Figura $5 \mathrm{~b}$ ). Assim, o conjunto de operações foi projetado para extrair e melhorar o
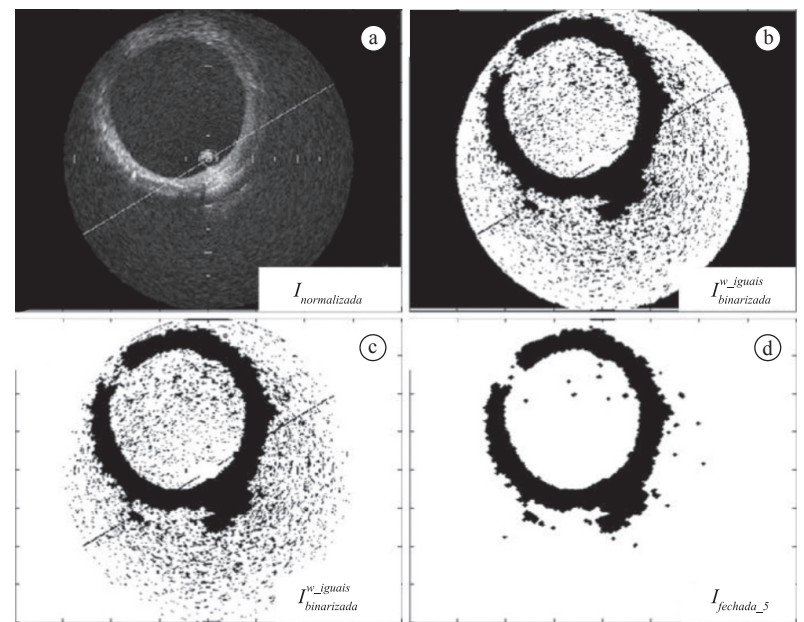

(c). (d)

(d)
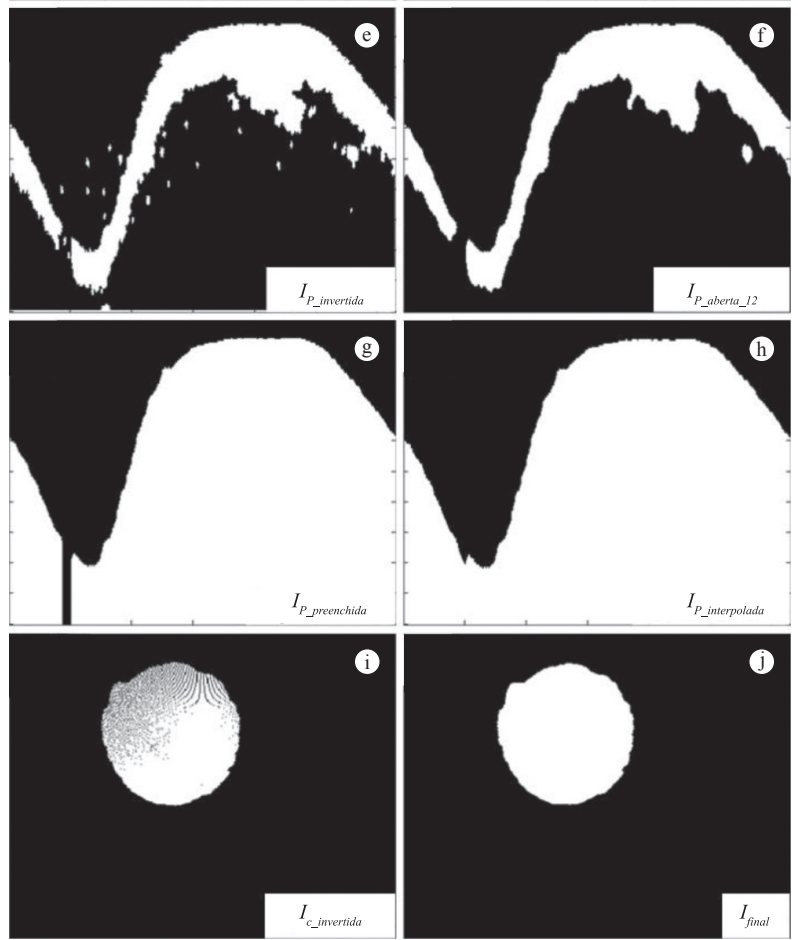

Figura 5. a) Imagem normalizada com “Branch opening”. b) Imagem binarizada para pesos iguais com “Branch Opening”. c) Imagem binarizada Sem Área Externa. d) Imagem fechada com kernel de raio $\mathrm{R}=5$. e) Imagem transformada a polar e invertida. f) Imagem após do fechamento com kernel de raio $\mathrm{R}=9$ e aberta com kernel de raio $\mathrm{R}=12$. g) Imagem Preenchida descendente. h) Imagem polar após interpolação. i) Imagem transformada para cartesiana e invertida. j) Imagem final após fechamento com kernel de raio dinâmico $R_{\text {din }}$.

Figure 5. a) Normalized image with Branch opening b) Binary image with Branch Opening for weights with the same value. c) Binary image without external area. d) Closed image with kernel of radius $R=5$. e) The logic negation of the previous image in the polar coordinates. f) Image after a closing followed by an opening procedure with kernels of radius $R=9$, and $R=12$, respectively. $g$ ) Image after a downwards filling procedure. h) Polar image after interpolation. i) The logic negation of the previous image in the Cartesian coordinates. $j$ ) Final image after a closing procedure with kernel of dynamic radius $R_{d i n}$ 
lúmen. Portanto, primeiramente, para evitar problemas futuros com algumas operações, a área externa da $I_{\text {binarizada }}^{\text {afinade }}$, (Figura 5b), é binarizada, resultando na imagem auxiliar $I_{\text {binarizada }}^{\text {SAE }}$ (Figura 5c). Segundo, por causa de ruídos trazidos pelas características das imagens IOCT e das operações de Fuzzy connectedness e binarização, sobre $I_{\text {binarizada }}^{\text {SAE }}$ (Figura $5 \mathrm{c}$ ) é feito um fechamento, (Tabela 1 - linha 4), com um elemento estruturante circular de raio $\mathrm{R}=5, S_{5}$, resultando na imagem auxiliar $I_{\text {fechada_5 } 5}$ (Figura 5d). Posteriormente, devido ao fato que a coronária e o lúmen tendem a serem circulares, o complemento da imagem $I_{\text {fechada_5 }}$ (Figura 5d), é transformada para coordenada polar, (Tabela 1 - linha 6), obtendo assim a imagem auxiliar $I_{p \_ \text {invertida }}$ (Figura 5e). Esse domínio foi adotado, pois torna as operações mais simples (Gil et al., 2006; Moraes e Furuie, 2011; Unal et al., 2008). Em quarto, para remover os ruídos brancos remanentes é feita sobre a imagem $I_{p_{-} \text {invertida }}$ (Figura 5e) uma combinação de fechamento, (Tabela 1 - linha 7), com um elemento estruturante circular de raio $\mathrm{R}$ $=8, S_{8}$, e uma abertura (Gonzalez e Woods, 2008), (Tabela 1 - linha 8), com um elemento estruturante circular de raio $\mathrm{R}=12, S_{12}$, resultando na imagem $I_{p_{\text {_aberta } 12}}($ Figura 5f). Em seguida, para isolar a área correspondente ao lúmen e ao "Branch Opening", sobre a imagem $I_{\text {p_aberta_l2 }_{12}}$ (Figura 5f), é realizada uma operação de preenchimento descendente, (Tabela 1 - linha 9), gerando a imagem $I_{p \text { preenchida }}$ (Figura 5g). Para remover a área correspondente ao "Branch Opening" e isolar a área do lúmen na imagem $I_{p \text { preenchida }}$ (Figura $5 \mathrm{~g}$ ) é realizada uma interpolação "spline" cúbica, (Tabela 1 - linha 10), sobre esta imagem, originando a imagem $I_{p \text { interpolada }}$ (Figura $5 \mathrm{~h}$ ). Finalmente, a imagem corrigida $I_{p \text { interpolada }}($ Figura $5 \mathrm{~h}$ ) é novamente invertida e trazida para coordenadas Cartesianas (Tabela 1 - linha 12), $I_{c \text { invertida }}$ (Figura 5i), e um último fechamento sobre esta, (Tabela 1 - linha 13), com um elemento estruturante circular, cujo raio é ajustado dinamicamente, $R_{d i n}$, é aplicado para tirar ruídos e melhorar as bordas do Lúmen final, gerando assim a imagem $I_{\text {final }}$ (Figura 5j).

\section{Resultados}

Para avaliar o método foram usadas 130 imagens de IOCT de dois humanos, três porcos e um coelho, fazendo desse, um conjunto de imagens desafiadoras, pois oferecem diferentes características, como a parede da coronária e lúmen de diferentes tamanhos, forma e posição espacial. Todas imagens foram adquiridas do Instituto do Coração do Hospital das Clínicas da Faculdade de Medicina da Universidade de São Paulo (InCor). O protocolo animal de estudo foi aprovado pelo comitê de ética, e os pacientes humanos assinaram o consentimento informado. A aquisição foi feita com pullback de $0,5 \mathrm{~mm} / \mathrm{s}$ e $20 \mathrm{f} / \mathrm{s}$ realizadas por um cateter de LightLab ImageWire unido a um sistema de imagem OCT (LightLabTM Optical Coherence Tomography LightLab Imaging, Inc., U.K). O custo computacional foi baseado num computador pessoal DELL com um processador Intel Core 2 Duo, $2.53 \mathrm{GHz}, 4 \mathrm{~GB}$ de RAM, Windows 7 a 32 bits e ImageJ sem otimização de código. Todas as imagens foram segmentadas e posteriormente comparadas com segmentações manuais "Gold Standards" feitas por especialistas. Na Figura 6 pode ser visto alguns resultados do método Fuzzy connectedness com afinidade Bhattacharyya proposto neste trabalho e seus respectivos "Gold Standards".

A avaliação da acurácia foi feita a partir do procedimento proposto por Udupa et al. (2006), o qual consiste no cálculo da média e desvio padrão das métricas "Verdadeiro Positivo" VP, "Falso Positivo" FP e "Falso Negativo" FN. A Tabela 2 resume as métricas e o custo computacional médio. Com pode ser observado na Tabela 2, os resultados obtidos mostraram uma alta acurácia para as três funções de afinidades usadas, com um VP em torno de $98 \%$ e um FP próximo de $2 \%$. Além das métricas acima além dois outros índices foram usados, Overlap Dice (Dice, 1945) e Overlap (Kupinski e Giger, 1998), assim os resultados obtidos pudessem ser comparados com estudos publicados. Para as duas métricas, e diferentes funções de afinidades, uma acurácia próxima de $96 \%$, e $98 \%$, foi obtida, em ambos os casos são melhores que as obtidas pela literatura (Dice, 1945; Kupinski e Giger, 1998). O custo computacional em

Tabela 2. Resultados da Acurácia.

Table 2. Results of Accuracy.

\begin{tabular}{ccccccr}
\hline & VP (\%) & FP (\%) & FN (\%) & Overlap (\%) & Dice (\%) & $\begin{array}{c}\text { Custo } \\
\text { (segundos) }\end{array}$ \\
\hline \multirow{2}{*}{ Pesos iguais } & 98,54 & 3,02 & 1,46 & 95,75 & 97,8 & 2,35 \\
& $\pm 1,47$ & $\pm 3,42$ & $\pm 1,47$ & $\pm 3,18$ & $\pm 1,72$ & $\pm 0,85$ \\
\hline \multirow{2}{*}{ Pesos Dinâmicos } & 97,97 & 2,19 & 2,03 & 95,95 & 97,90 & 2,18 \\
& $\pm 2,19$ & $\pm 3,06$ & $\pm 2,19$ & $\pm 3,30$ & $\pm 1,80$ & $\pm 0,66$ \\
\hline \multirow{2}{*}{ Bhattacharyya } & 97,73 & 1,82 & 2,27 & 96,03 & 97,95 & 2,96 \\
& $\pm 2,17$ & $\pm 2,20$ & $\pm 2,17$ & $\pm 3,08$ & $\pm 1,71$ & $\pm 0,75$ \\
\hline
\end{tabular}


torno de 2,5 segundos, por imagem segmentada, é muito mais rápido que manualmente (86 segundos) (Papadogiorgaki et al., 2008).

\section{Discussão}

O crescente uso de IOCT para diagnosticar doenças em coronárias faz que sejam desenvolvidos métodos computacionais que ajudem ao médico a tomar decisões.
Como foi discutido anteriormente, a literatura mostra alguns enfoques computacionais para a segmentação do lúmen na coronária em imagens IOCT (Dubuisson et al., 2009; Gurmeric et al., 2009; Sihan et al., 2008; Tsantis et al., 2012; Tung et al., 2011). Contudo, apesar dos esforços, a ausência de um número razoável de métodos, e resultados mais acurados dos mesmos, faz com que a procura de métodos alternativos de segmentação da coronária continue.
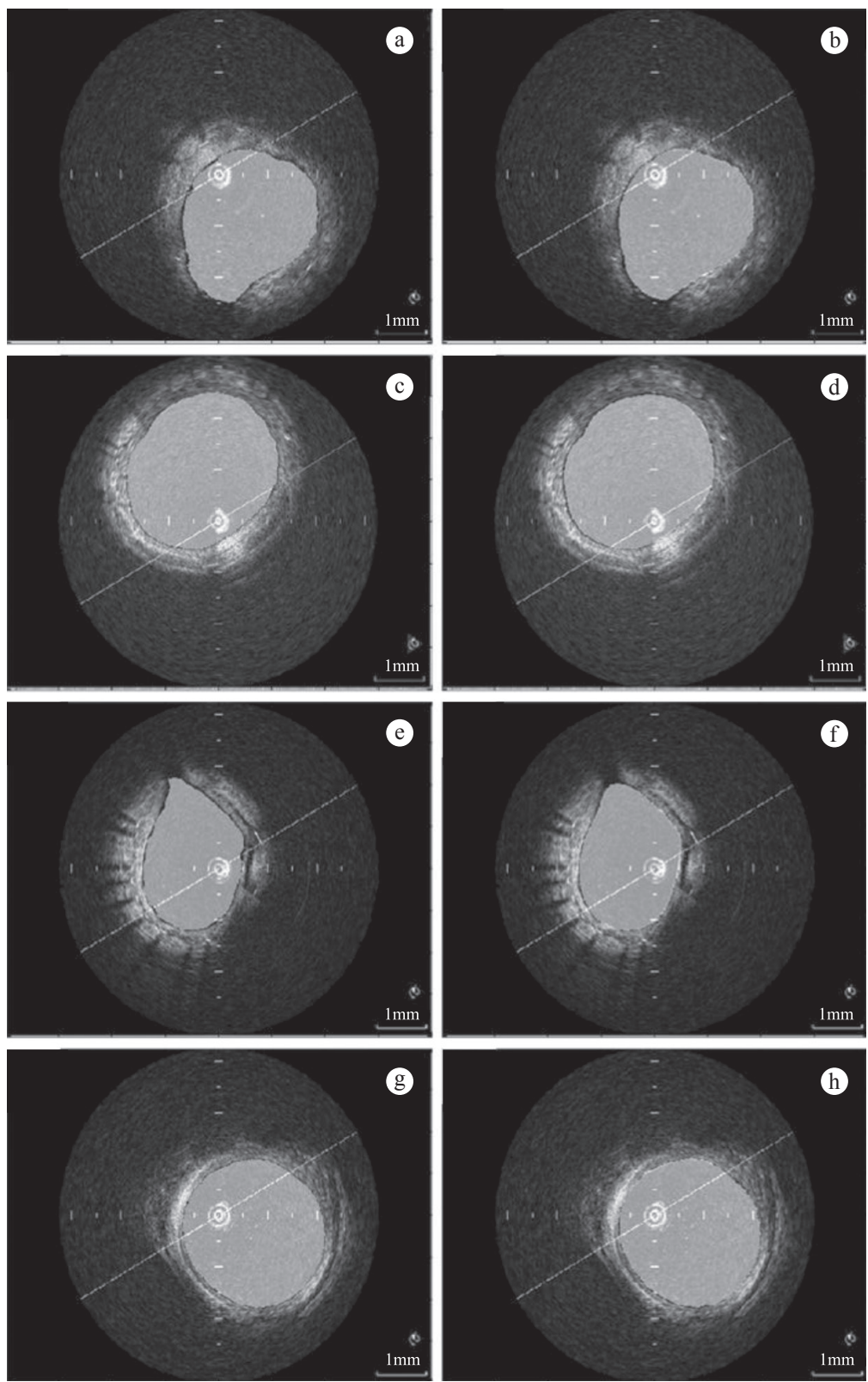

Figura 6. (a), (c), (e) e (g) Imagens com lúmen segmentado feitos com afinidade Bhattacharyya proposto neste trabalho e (b), (d), (f) e (h) seus respectivos "Gold Standard".

Figure 6. (a), (c), (e) and (g) Segmented images using Bhattacharyya as affinity function. proposed by this paper, and $(b),(d),(f)$ and $(h)$ their corresponding "Gold Standard". 
A metodologia apresentada neste trabalho para a segmentação do lúmen pode ser divida em três etapas. A primeira, Pré-processamento, é feita com a finalidade de remover atributos não desejados da imagem original. A segunda, Processamento, é utilizado o método de Fuzzy Connectedness, nunca antes utilizado para segmentação do lúmen na coronária, em conjunto com um novo método de binarização, para retirar informações do lúmen. Por último, no Pós-processamento, é feito uma combinação de múltiplas operações morfológicas, para assim, obter com precisão o objeto do lúmen.

Como pode ser visto na Tabela 2, os métodos, em todas as métricas, apresentam bons resultados com respeito à acurácia. Observando a tabela, também pode se notar que os métodos apresentam desempenhos similares, tanto na média como nos desvios padrões entre eles. Contudo, o método que utiliza afinidade Bhattacharyya apresenta menor FP e possui a vantagem de ser mais robusto, pois esse, não utiliza somente características da intensidade dos pixels (c, d), mais também características da região vizinha a cada pixel com seu kernel. Comparando com a literatura, Tsantis et al. (2012) oferece um método de segmentação automática do lúmen para imagens da artéria femoral com um valor de overlap de $93,7 \pm 4,5$, eficácia quase $3 \%$ inferior ao da nossa proposta. Em Tung et al. (2011) a segmentação automática do lúmen de coronárias é feita obtendo um valor médio de Overlap DICE de 97,25 $\pm 2,0$, o qual é relevante, mas ainda inferior ao obtido neste trabalho. Finalmente, na comparação com os métodos já publicados, podemos ver que o método proposto aqui apresentou resultados melhores do que os da literatura. Levando-se em conta as dificuldades das imagens por serem de diferentes fontes, a acurácia e robustez do método são constatadas, oferecendo assim uma nova alternativa semiautomática de realizar a segmentação do lúmen.

\section{Conclusão}

Em resumo, a boa acurácia e robustez, constatado pelos resultados, sendo superiores a métodos publicados, corrobora a eficácia das técnicas e encoraja seu uso. As principais contribuições deste trabalho são: (i) a utilização de Fuzzy Connectedness para segmentação de imagens IOCT, (ii) a utilização do coeficiente de Bhattacharyya para fazer uma nova relação de afinidade para Fuzzy Connectedness, (iii) uma nova forma de binarização, que aproveita o máximo das informações extraídas pelas imagens de saída do Fuzzy Connectedness, (iv) um método simples para a detecção do "Branch Opening", (v) um conjunto de operações morfológicas, projetadas para três diferentes funções de afinidades, e por último, (vi) a combinação do Fuzzy Connectedness com operações morfológicas para atingir com eficácia a segmentação do objeto.

Finalmente, o fato de que o método de Fuzzy Connectedness seja semiautomático pode ser visto como uma limitação para a técnica. Portanto, para trabalhos futuros, serão investigadas alternativas para que o usuário só precise implantar a semente na primeira imagem, e a partir dessa imagem, o algoritmo, se torne automático. Em segundo, métodos mais robustos de binarização para redução de ruídos na imagem binarizada serão testados. Adicionalmente, serão criadas novas relações locais de afinidades para Fuzzy Connectedness, e seus desempenhos serão comparados com as atuais.

\section{Agradecimentos}

CNPq (Conselho Nacional de Ciência e Tecnologia - Brasil), FAPESP (Fundação de Amparo à Pesquisa do Estado de São Paulo), InCor (Instituto do Coração do Hospital das Clínicas da Faculdade de Medicina da Universidade de São Paulo - Brasil), LEB-USP (Laboratório de Engenharia Biomédica da Universidade de São Paulo).

\section{Referências}

Aherne F, Thacker N, Rockett P. The Bhattacharyya metric as an absolute similarity measure for frequency coded data. Kybernetika. 1998; 32(4):1-7.

Barajas J, Caballero K, Rodrigues O, Radeva P. Cardiac phase extraction in IVUS sequences using 1-D Gabor filters. In: Annual International Conference Of The IEEE: Proceedings of the 29th Annual International Conference Of The IEEE; 2007; Lyon. EMBS; 2007. p. 343-6 PMid:18001960.

Besag J. On the statistical analysis of dirty pictures. Journal of the Royal Statistical Society. Series B (Methodological). 1986; 48:259-302.

Bhattacharyya A. On a measure of divergence between two statistical populations defined by their probability distribution. Bulletin of the Calcutta Mathematical Society. 1943; 35:99-110.

Bouma BE, Tearney GJ, Yabushita H, Shishkov M, Kauffman CR, Gauthier DD, MacNeill BD, Houser SL, Aretz HT, Halpern EF, Jang IK. Evaluation of intracoronary stenting by intravascular optical coherence tomography. Heart. 2003; 89:317-21. PMid:12591841 PMCid:1767586. http://dx.doi.org/10.1136/heart.89.3.317

Boykov YY, Jolly MP. Interactive graph cuts for optimal boundary \& region segmentation of objects in N-D images. In: IEEE International Conference on Computer Vision: Proceedings of the IEEE International Conference on Computer Vision; 2001 July 7-14; Vancouver, Canada. Vancouver; 2001. p. 105-12. 
Brezinski M. Optical Coherence Tomography: Principles and Applications. Academic Press; 2006.

Canny J. A computational approach to edge detection. IEEE Transactions on Pattern Analysis and Machine Intelligence. 1986; 8:679-98. PMid:21869365. http://dx.doi. org/10.1109/TPAMI.1986.4767851

Ciesielski KC, Udupa JK, Saha PK, Zhuge Y. Iterative relative fuzzy connectedness for multiple objects with multiple seeds. Computer Vision and Image Understanding. 2007; 107:16082. PMid:18769655 PMCid:2442428. http://dx.doi. org/10.1016/j.cviu.2006.10.005

Costa RFB, Fagundes DJ. Modelos experimentais de hiperplasia intimal: efeitos da radiação ionizante. Acta Cirurgica Brasileira. 2002; 17(3):25-30. http://dx.doi. org/10.1590/S0102-86502002000300007

Dempster AP, Laird NM, Rubin DB. Maximum Likelihood from Incomplete Data via the EM Algorithm. Journal of the Royal Statistical Society. Series B (Methodological). 1977; 39(1):1-38.

Dice LR. Measures of the amount of ecologic association between species. Ecology. 1945; 26:297-302. http://dx.doi. org/10.2307/1932409

Dougherty G. Digital Image Processing for Medical Applications. Cambridge University Press; 2009.

Dubuisson F, Kauffmann C, Motreff P, Sarry L. In vivo oct coronary imaging augmented with stent reendothelialization score. Medical Image Computing and Computer-Assisted Intervention. 2009; 5761:475-82.

Gil D, Hernandez A, Rodriguez O, Mauri J, Radeva P. Statistical strategy for anisotropic adventitia modelling in IVUS. IEEE Transactions on Medical Imaging. 2006; 25(6):768-78. PMid:16768241. http:// dx.doi.org/10.1109/TMI.2006.874962

Gonzalez RC, Woods RE. Digital Image Processing. 3rd ed. New Jersey: Pearson Prentice-Hall; 2008.

Griñó C, Lugo F, León M, Ligero S, Ruiz JM, Montero J. Tomografía de Coherencia Óptica (OCT) Funcionamiento y utilidad en patología macular (I). Gaceta Óptica. 2008; 12-4.

Gurmeric S, Isguder GG, Carlier S, Unal G. A new 3-d automated computational method to evaluate in-stent neointimal hyperplasia in in-vivo intravascular optical coherence tomography pullbacks. Medical Image Computing and Computer-Assisted Intervention. 2009; 5762:776-85.

Kass M, Witkin A, Terzopoulos D. Snakes: Active contour models. International Journal of Computer Vision. 1988; 1(4):321-31. http://dx.doi.org/10.1007/ BF00133570

Kupinski MA, Giger ML. Automated seeded lesion segmentation on digital mammograms. IEEE Transactions on Medical Imaging. 1998; 17:510-17. PMid:9845307. http://dx.doi.org/10.1109/42.730396

Meng L, Lu B, Zhang S, Yv B. In vivo optical coherence tomography (OCT) of experimental thrombosis in a rabbit carotid model. Heart. 2007; 24:777-80.
Moonis G, Liu J, Udupa JK, Hackney DC. Estimation of tumor volume with fuzzy connectedness segmentation of MR Images. American Journal of Neuroradiology. 2002; 23:356-63. PMid:11900999.

Moraes MC, Furuie SS. An approach to automatically segment the media-adventitia borders in IVUS Revista Brasileira de Engenharia Biomédica. 2010; 26(3):219-33.

Moraes MC, Furuie SS. Automatic coronary wall segmentation in intravascular ultrasound images using binary morphological reconstruction. Ultrasound in Medicine \& Biology. 2011; 37:1486-99. PMid:21741157. http://dx.doi. org/10.1016/j.ultrasmedbio.2011.05.018

Otsu N. A threshold selection method from gray-scale histogram. IEEE Transactions on Systems, Man and Cybernetics. 1979; 9(1): 62-6. http://dx.doi.org/10.1109/ TSMC.1979.4310076

Papadogiorgaki M, Mezaris V, Chatzizisis YS, Giannoglou GD, Kompatsiaris I. Image analysis techniques for automated ivus contour detection. Ultrasound in Medicine \& Biology 2008; 34(9):1482-98.

Pednekar A, Kakadiaris IA. Image segmentation based on fuzzy connectedness using dynamic weights. IEEE Transactions on Image Processing. 2006; 15:1555-62. http:// dx.doi.org/10.1109/TIP.2006.871165

Pollock ML, Wilmore, JH. Exercícios na saúde e na doença: avaliação e prescrição para prevenção e reabilitação. 2. ed. Rio de Janeiro: Medsi; 1993.

Puri R, Worthley MI, Nicholls SJ. Intravascular Imaging of Vulnerable Coronary Plaque: Current and Future Concepts. Nature Reviews Cardiology. 2011; 8(3):131-39. http:// dx.doi.org/10.1038/nrcardio.2010.210

Rieber J, Meissner O, Babaryka G, Oswald SRM, Koenig A, Schiele, T, Shapiro M, Theisen K, Reiser M, Klauss V, Hoffmann U. Diagnostic Accuracy of Optical Coherence Tomography and Intravascular Ultrasound for the Detection and Characterization of Atherosclerotic Plaque Composition In Ex-Vivo Coronary Specimens: A Comparison With Histology. Coronary Artery Disease. 2006; 17(5):425-30. PMid:16845250. http://dx.doi.org/10.1097/00019501200608000-00005

Rubinstein M, Schalch P, Di Silvio M, Betancourt MA, Wong BJ. Optical coherence tomography applications in otolaryngology. Acta Otorrinolaringologica Espanola, 2009; 60(5):357-63. http://dx.doi.org/10.1016/ S2173-5735(09)70157-7

Sihan K, Botka C, Post F, De Winter S, Regar E, Hamers $\mathrm{R}$, Bruining N. A novel approach to quantitative analysis of intravascular optical coherence tomography imaging. Computers in Cardiology; 2008; 1089-92.

Souza AFL. Abordagens para a segmentação de coronárias em ecocardiografia [dissertação]. São Paulo: Universidade de São Paulo; 2010.

Souza AJN, Nascimento AJL, Carvalho AS. Reestenose Intrastent. Opções Terapêuticas na Realidade Brasileira. Revista Brasileira de Cardiologia Invasiva. 2004; 12(4):193-202. 
Tsantis S, Kagadis G, Katsanos K, Karnabatidis D, Bourantas G, Nikiforidis G. Automatic vessel lumen segmentation and stent strut detection in intravascular optical coherence tomography. Medical Physics. 2012; 39:503-13. PMid:22225321. http://dx.doi.org/10.1118/1.3673067

Tung KP, Shi W, De Silva R, Edwards E, Rueckert D. Automatical vessel wall detection in intravascular coronary OCT. In: IEEE International Symposium on Biomedical Imaging: Proceedings of the IEEE International Symposium on Biomedical Imaging; 2011 Mar 30-Apr 02; Chicago. Chicago; 2011. p. 610-3.

Unal G, Bucher S, Carlier S, Slabaugh G, Fang T, Tanaka K. Shape-driven segmentation of the arterial wall in intravascular ultrasound images. IEEE Transactions on Information Technology in Biomedicine. 2008; 12:335-47. PMid:18693501. http://dx.doi.org/10.1109/TITB.2008.920620

Unal G, Gurmeric S, Carlier SG. Stent Implant FollowUp in Intravascular Optical Coherence Tomography Images. International Journal of Cardiovascular Imaging. 2010; 26(7):809-16. PMid:19777367. http:// dx.doi.org/10.1007/s10554-009-9508-4

Udupa JK, LeBlanc VR, Zhuge Y, Imielinska C, Schmidt H, Currie LM, Hirsch BE, Woodburn J. A Framework for Evaluating Image Segmentation Algorithms. Computerized
Medical Imaging and Graphics. 2006; 30(2):7587. PMid:16584976. http://dx.doi.org/10.1016/j. compmedimag.2005.12.001

Udupa JK, Samarasekera S. Fuzzy connectedness and object definition: theory, algorithms, and applications in image segmentation. Graphical Models and Image Processing. 1996; 58(3): 246-61. http://dx.doi.org/10.1006/ gmip.1996.0021

Udupa JK, Saha PK. Fuzzy connectedness and image segmentation. Proceedings of the IEEE. 2003; 91:1649-69. http://dx.doi.org/10.1109/JPROC.2003.817883

World Health Organization - WHO. Global status report on noncommunicable diseases 2010. WHO; 2011a. Available from: http://www.who.int/nmh/publications/ncd_report2010/ en/

World Health Organization - WHO. Nomcommunicable diseases and mental healt. WHO; 2011b. Available from: http://www.who.int/nmh/countries/bra_en.pdf

Zaidi H, Naqa EI. PET-guided delineation of radiation therapy treatment volumes: a survey of image segmentation techniques. European Journal of Nuclear Medicine and Molecular Imaging. 2010; 37:1-37. PMid:20336455. http:// dx.doi.org/10.1007/s00259-010-1423-3

\footnotetext{
Autores

Diego Armando Cardona Cardenas*, Matheus Cardoso Moraes, Sérgio Shiguemi Furuie

Laboratório de Engenharia Biomédica, Departamento de Engenharia de Telecomunicações e Controle, Escola Politécnica, Universidade de São Paulo - USP, Av. Professor Luciano Gualberto, Travessa 3, 158, CEP 05508-970, São Paulo, SP, Brasil.
} 